\title{
Modelos de Urna: \\ Um Estudo de Tempos de Espera via Processo de Poisson
}

\author{
Dario Nery
}

DISSERTAÇÃO APRESENTADA

$\mathrm{AO}$

INSTITUT, DE MATEMÁTICA E ESTATÍSTICA

DA

UNIVERSIDADE DE SÃO PAULO

PARA OBTENÇÁO DO GRAU DE MESTRE

EM

ESTATÍSTICA

Área de Concentração: Probabilidade

Orientador: Prof. Dr. Flávio Wagner Rodrigues

-São Paulo, Junho de 1992- 
A Danielle

A Elaine

Aos meus pais

A Tia Laide 


\section{AGRADECIMENTOS}

A todos que colaboraram neste trabalho. Em especial

- Prof. Flávio Wagner Rodrigues - pela sugestào do tema e orientaçào.

- Prof. Sérgio Wechsler - pelas valiosas sugestòes no decorrer do trabalho e grande amigo.

- Rinaldo Artes - pela amizade e incentivo desde os tempos de graduação.

- Fábio Prates Machado - grande amigo em todas as ocasiòes.

- Aos colegas de mestrado.

- Aos colegas do Departamento de Estatística. em particular aos Profs. Adilson Simonis e Antonio Carlos Pedroso de Lima. 


\begin{abstract}
Some occupancy problems are studied by considering several ways by which balls may be distributed. The waiting time until a certain condition is met is then studied. A solution introduced by Holst (1986) changing the discrete waiting time to continuous time through embeddment into a Poisson Process is constructed. Applications to Brazilian lotteries numbers are presented.
\end{abstract}

\title{
RESUMO
}

Neste trabalho estudamos alguns problemas de ocupação considerando a maneira como as bolas são distribuídas. O tempo de espera até que determinada condição esteja satisfeita tambêm é estudado. É desenvolvida uma proposta de Holst (1986) transformando o problema de tempo discreto em contínuo, através de uma imerssão em um Processo de Poisson. Aplicações às loterias de números Loto e Sena são apresentadas. 


\section{Conteúdo}

1 O Problema Clássico da Ocupação.

1.1 Introduçào . . . . . . . . . . . . . . . 2

1.2 Modelo Parcialmente Aleatório para Bolas Idênticas. . . . . . . . . 7

1.2.1 Distribuiçào do número de urnas ocupadas e do número de urnas vazias 7

1.2 .2 Momentos de $M_{0}^{r}$ e de $M_{v c}^{r} \ldots \ldots \ldots \ldots \ldots \ldots$

1.2 .3 Aplicaçào . . . . . . . . . . . . . . . . 11

1.3 Modelo Totalmente Aleatório. . . . . . . . . . . . . . . 16

1.3.1 Distribuiçào do número de urnas ocupadas e do número de urnas vazias 18

1.3.2 Momentos de $M_{0}^{r}$ e de $M_{u c}^{r} \ldots \ldots \ldots \ldots \ldots . \ldots . \ldots 19$

1.3.3 Distribuiçào e esperança do número de urnas com exatamente $k$ bolas . 20

2 O Problema das Comissões. 
2.1 Introdução . . . . . . . . . . . . . . . . . . . . . . . . 24

2.2 Distribuição e Momentos do Numero de Bolas que Faltam Sair . . . . . . . . . 25

3 Problemas de Tempo de Espera.

3.1 Introdução . . . . . . . . . . . . . . . . . . . . . . . . . . . 29

3.2 Tempo de Espera no Problema da Ocupaçào. . . . . . . . . . . . . . . . . 30

3.2.1 O Problema do Colecionador . . . . . . . . . . . . . . . . 30

3.2 .2 Imersào em um Processo de Poisson . . . . . . . . . . . . . . . . . 33

3.2 .3 Aplicaçòes . . . . . . . . . . . . . . . . . . . . 35

3.3 Tempo de Espera no Problema das Comissòes. . . . . . . . . . . . . . . . . . . 39

3.3 .1 Aplicaçào. . . . . . . . . . . . . . . . . 4 41

3.3.2 Conclusòes e Comentários. . . . . . . . . . . . . . . 42

A Operador Diferença e Números de Stirling. $\quad 43$ 
Capítulo 1

O Problema Clássico da Ocupação. 


\subsection{Introdução}

Considere $N$ urnas numeradas de 1 a $N$ e um conjunto de $r$ bolas que serão distribuidas entre essas urnas. O problema clássico da ocupação consiste em determinar a distribuiçào de probabilidades do número de urnas ocupadas (ou do número de urnas vazias) após as $r$ bolas terem sido distribuídas. Uma maneira alternativa de se ver esse problema é considerar uma urna contendo $N$ bolas numeradas da qual retiramos $r$ bolas com reposição. Neste caso, nosso interesse é a distribuição do número de bolas distintas na amostra (ou do número de bolas que nào apareceram nenhuma vez). Dependendo da conveniência utilizaremos, ao longo deste trabalho, uma ou outra abordagem.

Johnson \& Kotz (1977) e Feller (1968) tratam do problema considerando duas situações diferentes: bolas distintas e bolas idênticas. Porém essa distinçào só faz sentido se levarmos em conta o processo de distribuiçào das bolas nas urnas.

Neste trabalho, duas maneiras de distribuir-se as bolas nas urnas serào consideradas.

Definição 1.1 Diremos que a distribuição é Totalmente Aleatória se para cada bola escolhermos independentemente e com igual probabilidade a urna onde deveremos colocá-la.

Definição 1.2 Chamamos Configuração a qualquer possivel distribuição das $r$ bolas nas $N$ urnas.

Definição 1.3 Diremos que a distribuiçào é Parcialmente Aleatória quando escolhermos a configuraçâo final das $r$ bolas nas $N$ urnas com igual probabilidade.

Desta maneira, temos 4 situaçòes conforme esquematizamos abaixo:

\begin{tabular}{l|c|c}
\hline \multirow{2}{*}{ Distribuição } & \multicolumn{2}{|c}{ Bolas } \\
\cline { 2 - 3 } $\begin{array}{l}\text { Totalmente } \\
\text { Aleatória }\end{array}$ & Distintas & Idênticas \\
\hline $\begin{array}{l}\text { Parcialmente } \\
\text { Aleatória }\end{array}$ & $\mathrm{B}$ & $\mathrm{C}$ \\
\hline
\end{tabular}


Exemplo 1.1 Para $r=3$ e $N=2$,

Figura 1 - Distribuição de 3 bolas distintas.
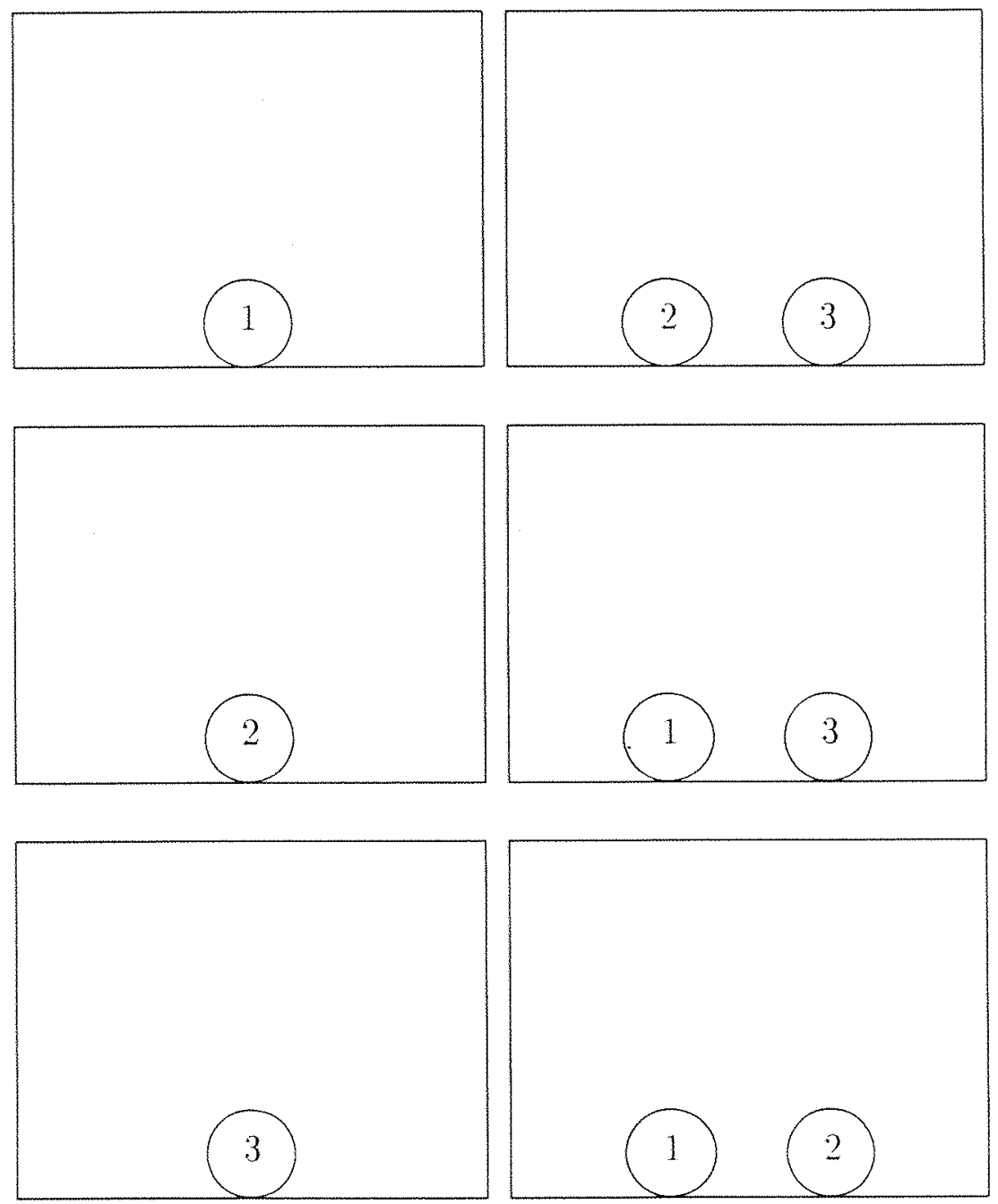

representam três configuraçòes distintas nas situaçôes $\mathrm{A}$ e $\mathrm{B}$; enquanto que nas situaçōes $\mathrm{C}$ e D, representam apenas a configuraçào seguinte 
Figura 2 - Distribuiçào de 3 bolas idênticas.
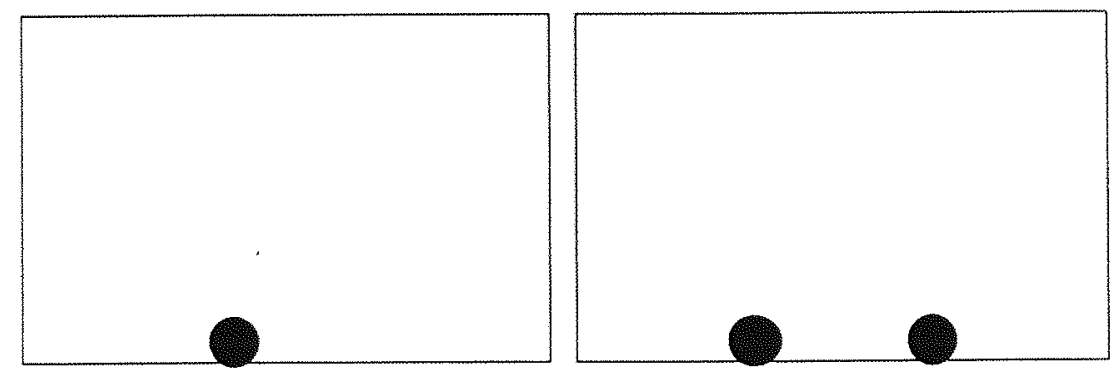

Situação A: Bolas distintas e distribuição Totalmente Aleatória.

Seja $B_{j}^{i}$ o evento bola $i$ na urna $j, i=1,2, \ldots, r ; j=1,2, \ldots, N$. Assim,

$$
P\left(B_{i}^{i}\right)=\frac{1}{N} . \quad i=1.2 \ldots . . j ;=1.2 \ldots N .
$$

Seja $C$ uma configuraçào qualquer. $C$ pode ser escrita em termos de $B_{j}^{i}$ da seguinte forma:

$$
C=B_{j_{1}}^{1} \cap B_{j_{2}}^{2} \bigcap \cdots \cap B_{j_{r}}^{r} \quad \operatorname{com} j_{i} \in\{1,2 \ldots, N\} \text { para } \quad i \in\{1,2, \ldots, r\}
$$

Entào,

$$
P(C)=\prod_{k=1}^{r} P\left(B_{j k}^{k}\right)=\left(\frac{1}{N}\right)^{r}=\frac{1}{N^{r}}
$$

Como para cada bola temos $N$ maneiras distintas de escolhermos uma urna, o número de configuraçôes distintas é $N^{r}$. Portanto, na situaçào A temos $N^{r}$ configuraçōes equiprováveis.

Situação B: Bolas distintas e distribuiçòo Parcialmente Aleatória.

Neste caso, também temos $N^{r}$ configuraçòes distintas que sào, por definiçào, equiprováveis. Logo, a probabilidade de qualquer configuraçào $C$ é:

$$
P(C)=\frac{1}{N^{r}}
$$


Situação C: Bolas idênticas e distribuiçào Totalmente Aleatória.

Neste caso, tudo se passa como se as bolas fossem numeradas porém sem que seus números sejam visiveis.

Seja $x_{j}$ o número de bolas alocadas na urna $j, j=1,2, \ldots, N$. Os número $x_{j}$ são chamados números de ocupação.

Cada configuraçào é então especificada pelo vetor $\vec{x}=\left(x_{1}, x_{2}, \ldots, x_{N}\right)$ e duas configuraçôes sào distintas se, e somente se. os vetores que as representam sào distintos.

Como o número total de bolas a serem distribuidas é $r$, temos:

$$
x_{1}+x_{2}+\cdots+x_{N}=r
$$

Portanto, o número de configurações distintas é igual ao número de soluções inteiras e não negativas da equação 1.1

Teorema 1.1 O número de maneiras distintas de se distribuir $r$ bolas identicas em $N$ urnas é dado por:

$$
\left(\begin{array}{c}
r+N-1 \\
r
\end{array}\right)=\left(\begin{array}{c}
r+N-1 \\
N-1
\end{array}\right)
$$

Prova: Representemos as bolas por $*$ e as $N$ urnas pelos $N$ espaços entre $N+1$ barras. Por exemplo, se tivermos $r=N=7$, a configuraçào

$$
|* *| *|*||| * * * \mid
$$

equivale a $x_{1}=2, x_{2}=x_{5}=x_{6}=0, x_{3}=x_{4}=1$ e $x_{7}=3$.

Para $r$ bolas e $N$ urnas temos entào $r+N+1$ símbolos. Como o primeiro e o último devem ser necessariamente barras restam $r+N-1$ simbolos sendo $r *$ e $N-1$ | para serem alocados. Observe que a distribuiçào fica completamente determinada quando escolhemos os lugares dos $r *$ ou das $N-1$ |. Consequentemente. o número total de distribuiçòes distintas é dado por 1.2 .

Como, cada bola pode ser colocada em qualquer uma das $N$ urnas com probabilidade $\frac{1}{N}$ e independentemente das demais, o vetor $\vec{x}=\left(x_{1}, x_{2}, \ldots, x_{N}\right)$ possui distribuiçào Multinomial $\left(r, \frac{1}{N}, \frac{1}{N}, \cdots, \frac{1}{N}\right)$. 
Sendo cada configuração especificada pelo vetor $\vec{x}$, temos

$$
P(C)=\left(\begin{array}{c}
r \\
x_{1}, x_{2}, \cdots, x_{N}
\end{array}\right)\left(\frac{1}{N}\right)^{r}=\frac{\left(\begin{array}{c}
r \\
x_{1}, x_{2}, \cdots, x_{N}
\end{array}\right)}{N^{r}}
$$

Situação D: Bolas idênticas e distribuiçào Parcialmente Aleatória.

Neste caso, o número de configuraçòes distintas é ainda dado por 1.2 porém elas são, por definiçầo, equiprováveis. Portanto, temos $\left(\begin{array}{c}r+N-1 \\ r\end{array}\right)$ configurações com probabilidade $\frac{1}{\left(\begin{array}{c}r+N-1 \\ r\end{array}\right)}=\frac{1}{\left(\begin{array}{c}r+N-1 \\ N-1\end{array}\right)}$

\section{Observações:}

1. Como podemos observar no caso de bolas distintas, os modelos Totalmente Aleatório e Parcialmente Aleatório (situaçōes A e B respectivamente) são iguais. O mesmo nào ocorre no caso de bolas idênticas (situaçòes ( e D).

2. No caso de bolas idênticas e distribuiçào Totalmente Aleatória (situaçào C), o denominador da expressào 1.3 é $N^{r}$, como no caso de bolas distintas (situaçòes A e B). O numerador por sua vez pode ser obtido através da soma de configuraçóes distintas nas situaçōes $A$ e $B$, conforme visto nas figuras 1 e 2 .

Nos problemas de ocupaçào estamos interessados nos números de ocupação $x_{j}$ de cada urna e nào nos elementos que as compòem. Desta forma, o fato das bolas serem ou não distinguiveis será irrelevante no caso de distribuiçào Totalmente Aleatória, e no caso de bolas distintas as duas formas de distribuição resultam em modelos iguais. Desta forma, para o objetivo deste trabalho podemos considerar apenas dois casos: um que engloba as situações $\mathrm{A}$, $\mathrm{B}$ e C que chamaremos simplesmente de Modelo Totalmente Aleatório, e outro relativo a situaçào D que chamaremos de Modelo Parcialmente Aleatório para Bolas Idênticas. 


\subsection{Modelo Parcialmente Aleatório para Bolas Idên- ticas.}

Conforme vimos na situação $\mathrm{D}$, no modelo parcialmente aleatório para bolas idênticas as $\left(\begin{array}{c}r+N-1 \\ r\end{array}\right)$ configurações sào equiprováveis. Assim, para qualquer configuração $r$ temos,

$$
P(C)=\frac{1}{\left(\begin{array}{c}
r+N-1 \\
r
\end{array}\right)}
$$

Este modelo é conhecido na física como Estatística de Bose-Einstein e é utilizado para descrever o comportamento de fótons, núcleos e átomos que contenham um número par de particulas elementares (Margenau, H. \& Murphy. G. M. (1943)).

Sendo as configuraçòes equiprováveis, a probabilidade de um evento A qualquer é dada. por

$$
P(A)=\frac{\#(A)}{\left(\begin{array}{c}
r+N-1 \\
r
\end{array}\right)}
$$

onde \#(A) representa o número de configuraçòes que satisfazem o evento $A$.

\subsubsection{Distribuição do número de urnas ocupadas e do número de urnas vazias}

Teorema 1.2 O número de maneivas distintas de se alocar $r$ bolas idênticas em $N$ urnas de modo que nenhuma fique varia é dado por:

$$
\left(\begin{array}{c}
r-1 \\
N-1
\end{array}\right)
$$


Prova: Como as bolas são idênticas e queremos que todas as urnas tenham pelo menos uma bola cada, colocamos arbitrariamente uma bola em cada urna. Sobram então $r-N$ bolas para serem alocadas em $N$ urnas. Assim, por 1.2 o número de maneiras distintas é dado por

$$
\left(\begin{array}{c}
r-N+N-1 \\
N-1
\end{array}\right)=\left(\begin{array}{c}
r-1 \\
N-1
\end{array}\right)
$$

Lema 1.1 Seja $M_{j}^{r}$ o número de urnas com exatamente $j$ bolas, após as $r$ bolas terem sido alocadas, $j=0,1,2, \ldots, r \in M_{o c}^{r}$ o número de urnas ocupadas. Então

$$
\begin{gathered}
P\left(M_{o c}^{r}=k\right)=\frac{\left(\begin{array}{c}
N \\
k
\end{array}\right)\left(\begin{array}{l}
r-1 \\
k-1
\end{array}\right)}{\left(\begin{array}{c}
r+N-1 \\
r
\end{array}\right)} \cdot k=1,2 \ldots \min (N, r) \\
P\left(M_{0}^{r}=k\right)=\frac{\left(\begin{array}{c}
N \\
k
\end{array}\right)\left(\begin{array}{c}
r-1 \\
N-k-1
\end{array}\right)}{\left(\begin{array}{c}
r+N-1 \\
r
\end{array}\right)} \cdot k=\max (0, N-r), \ldots, N-1
\end{gathered}
$$

Prova: Observe que,

$$
\begin{aligned}
& M_{0}^{r}+M_{1}^{r}+M_{2}^{r}+\cdots+M_{r}^{r}=N \\
& M_{0 c}^{r}=M_{1}^{r}+M_{2}^{r}+\cdots+M_{r}^{r}=N-M_{0}^{r} .
\end{aligned}
$$

Para determinarmos a distribuiçào de $M_{0 \cdot}^{r}$. observemos que $M_{o c}^{r}=k$ se e somente se todas as $r$ bolas forem colocadas em exatamente $k$ urnas. com a restriçào de que nenhuma dessas $k$ urnas fiquem vazias.

Como as $k$ urnas podem ser escolhidas de $\left(\begin{array}{c}N \\ k\end{array}\right)$ maneiras distintas e por 1.4 o número de 
maneiras de se distribuir $r$ bolas em $k$ urnas de modo que nenhuma fique vazia é $\left(\begin{array}{c}r-1 \\ k-1\end{array}\right)$, temos

$$
P\left(M_{o c}^{r}=k\right)=\frac{\left(\begin{array}{c}
N \\
k
\end{array}\right)\left(\begin{array}{c}
r-1 \\
k-1
\end{array}\right)}{\left(\begin{array}{c}
r+N-1 \\
r
\end{array}\right)}
$$

e consequentemente por 1.7 e 1.5

$$
P\left(M_{0}^{r}=k\right)=P\left(M_{o c}^{r}=N-k\right)=\frac{\left(\begin{array}{c}
N \\
k
\end{array}\right)\left(\begin{array}{c}
r-1 \\
N-k-1
\end{array}\right)}{\left(\begin{array}{c}
r+N-1 \\
r
\end{array}\right)}
$$

1.2.2 Momentos de $M_{0}^{r}$ e de $M_{o c}^{r}$

Lema 1.2 Seja $\mu_{M_{0}^{r}}^{(i)}$ o i-ésimo momento fatorial de $M_{0}^{r}$. isto é,

$$
\mu_{M_{0}^{r}}^{(i)}=\mathrm{E}\left(M_{0}^{r(i)}\right)=\mathrm{E}\left[M_{0}^{r}\left(M_{0}^{r}-1\right) \cdots\left(M_{0}^{r}-i+1\right)\right]
$$

Entäo, para $i \leq N-1$

$$
\mu_{M_{0}^{r}}^{(i)}=\frac{N^{(i)}\left(\begin{array}{c}
r+N-i-1 \\
r
\end{array}\right)}{\left(\begin{array}{c}
r+N-1 \\
r
\end{array}\right)}
$$


Prova: Para $i \leq N-1$, temos

$$
\begin{aligned}
& \mu_{M_{0}^{r}}^{(i)}=\mathbf{E}\left(M_{0}^{r(i)}\right)=\sum_{k=0}^{N-1} k^{(i)} P\left(M_{0}^{r}=k\right)=\sum_{k=0}^{N-1} k^{(i)} \frac{\left(\begin{array}{c}
N \\
k
\end{array}\right)\left(\begin{array}{c}
r-1 \\
N-k-1
\end{array}\right)}{\left(\begin{array}{c}
r+N-1 \\
r
\end{array}\right)} \\
& =\sum_{k=0}^{N-1} k(k-1) \ldots(k-i+1) \frac{N !}{k !(N-k) !} \frac{\left(\begin{array}{c}
r-1 \\
N-k-1
\end{array}\right)}{\left(\begin{array}{c}
r+N-1 \\
r
\end{array}\right)} \\
& =\sum_{k=i}^{N-1} \frac{N !}{(k-i) !(N-k) !} \frac{\left(\begin{array}{c}
r-1 \\
N-k-1
\end{array}\right)}{\left(\begin{array}{c}
r+N-1 \\
r
\end{array}\right)} \\
& =N^{(i)} \sum_{k=i}^{N-1}\left(\begin{array}{c}
N-i \\
k-i
\end{array}\right) \frac{\left(\begin{array}{c}
r-1 \\
N-k-1
\end{array}\right)}{\left(\begin{array}{c}
r+N-1 \\
r
\end{array}\right)} \\
& =N^{(i)} \frac{\sum_{j=0}^{N-i-1}\left(\begin{array}{c}
N-i \\
j
\end{array}\right)\left(\begin{array}{c}
r-1 \\
N-i-j-1
\end{array}\right)}{\left(\begin{array}{c}
r+N-1 \\
r
\end{array}\right)}
\end{aligned}
$$

Mas o numerador de 1.9 é

$$
\sum_{j=0}^{N-i-1}\left(\begin{array}{c}
N-i \\
j
\end{array}\right)\left(\begin{array}{c}
r-1 \\
N-i-j-1
\end{array}\right)=\left(\begin{array}{c}
r+N-i-1 \\
r
\end{array}\right)
$$

o que conclui a prova. 
Lema 1.3 A esperança e a variância de $M_{0}^{r} \epsilon$ de $M_{o c}^{r}$ sâo dadas por:

$$
\begin{aligned}
\mathbf{E}\left(M_{0}^{r}\right) & =\frac{N(N-1)}{r+N-1} \\
\operatorname{Var}\left(M_{0}^{r}\right) & =\frac{N r(N-1)(r-1)}{(r+N-1)^{2}(r+N-2)} \\
\mathbf{E}\left(M_{o c}^{r}\right) & =N-\mathbf{E}\left(M_{0}^{r}\right)=N-\frac{N(N-1)}{r+N-1}=\frac{N r}{r+N-1} \\
\operatorname{Var}\left(M_{o c}^{r}\right) & =\operatorname{Var}\left(M_{0}^{r}\right)=\frac{N r(N-1)(r-1)}{(r+N-1)^{2}(r+N-2)}
\end{aligned}
$$

Prova: Para provar 1.10 e 1.11 basta ver que $\mathrm{E}\left(M_{0}^{r}\right)=\mu_{M_{0}^{r}}^{(1)}$ e $\operatorname{Var}\left(M_{0}^{r}\right)=\mu_{M_{0}^{r}}^{(2)}+\mu_{M_{0}^{r}}^{(1)}-\left[\mu_{M_{0}^{r}}^{(1)^{2}}\right]$ e aplicar 1.8.

No caso de 1.12 e de 1.13 , temos por 1.7 que $M_{o c}^{r}=N-M_{0}^{r}$. Logo por 1.10 e 1.11 temos

$$
\begin{aligned}
\mathbf{E}\left(M_{o c}^{r}\right) & =N-\mathbf{E}\left(M_{0}^{r}\right) \\
\operatorname{Var}\left(M_{o c}^{r}\right) & =\operatorname{Var}\left(M_{0}^{r}\right)
\end{aligned}
$$

\subsubsection{Aplicação}

Apesar de, na prática. nào conseguimos uma maneira de distribuirmos $r$ bolas em $N$ urnas que nos resulte no modelo visto acima. este pode ser útil na resoluçào de certos problemas. Apresentaremos aqui um problema que pode ser facilmente resolvido através do Modelo Parcialmente Aleatório.

A loteria de números Loto consiste de 100 dezenas numeradas de 00 a 99 das quais 5 sào sorteadas a cada concurso. Muitas pessoas se surpreendem ao verem que em vários concursos sào sorteadas dezenas consecutivas. Apesar de parecer, a primeira vista, estranho, este fato pode ser facilmente estudado através do Modelo Parcialmente Aleatório. 
Cada um dos possiveis resultados da Loto pode ser representado por uma sequência de 100 bolinhas, cada uma associada a uma dezena, sendo 5 bolinhas pretas (dezenas sorteadas) e 95 brancas (dezenas não sorteadas). Assim, se tivermos sorteadas as dezenas 04, 10, 16, 63 e 64 teremos a seguinte representação:

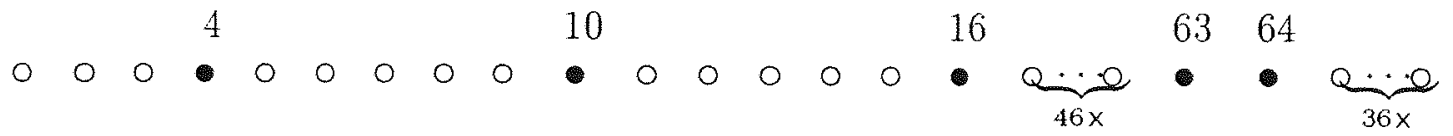

Consideremos entào cada bola preta (dezena sorteada) como sendo delimitador de uma urna. Assim, os espaços entre as 5 bolas pretas constituem 6 urnas e temos 95 bolas para serem distribuidas entre elas.

Como esses resultados sào equiprováveis, temos o Modelo Parcialmente Aleatório com $N=6$ urnas e $r=95$ bolas. Por 1.2 o número de possiveis resultados para um concurso da Loto é $\left(\begin{array}{c}95+6-1 \\ 6-1\end{array}\right)=\left(\begin{array}{c}100 \\ 5\end{array}\right)$ (como era de se esperar).

Ordenando-se as dezenas sorteadas em ordem crescente, seja $D_{i}$ a diferença entre a $i$-ésima e a $(i+1)$-ésima dezenas sorteadas, $i=1,2,3,4$ e seja $D=\min \left(D_{1}, D_{2}, D_{3}, D_{4}\right)$.

Então,

$$
D \geq y \leftrightarrow\left(D_{1} \geq y \cdot D_{2} \geq y \cdot D_{3} \geq y \cdot D_{4} \geq y\right)
$$

Mas $D_{i} \geq y$ se, e somente se, tivermos pelo menos $y-1$ bolas na urna $i+1, i=1,2,3,4$.

Restam entào $95-4(y-1)$ bolas para serem distribuidas entre as 6 urnas. Novamente por 1.2 , isso pode ser feito de $\left(\begin{array}{c}99-4 y+6-1 \\ 6-1\end{array}\right)=\left(\begin{array}{c}104-4 y \\ 5\end{array}\right)$ maneiras distintas. Logo,

$$
P(D \geq y)=\frac{\left(\begin{array}{c}
104-4 y \\
5
\end{array}\right)}{\left(\begin{array}{c}
100 \\
5
\end{array}\right)}
$$


Como $P(D=y)=P(D \geq y)-P(D \geq y+1)$, temos por 1.14 que:

$$
P(D=y)=\frac{\left(\begin{array}{c}
104-4 y \\
5
\end{array}\right)-\left(\begin{array}{c}
100-4 y \\
5
\end{array}\right)}{\left(\begin{array}{c}
100 \\
5
\end{array}\right)}, \quad 1 \leq y \leq 24
$$

A tabela abaixo apresenta esta distribuiçào.

Tabela 1.1 - Distribuição de Probabilidades da Diferença Mínima $D$ entre os números sorteados na Loto.

\begin{tabular}{|c|c||c|c|}
\hline $\mathrm{D}$ & Probabilidade & $\mathrm{D}$ & Probabilidade \\
\hline 1 & 0.1881 & 13 & 0.0118 \\
2 & 0.1587 & 14 & 0.0083 \\
3 & 0.1328 & 15 & 0.0057 \\
4 & 0.1103 & 16 & 0.0037 \\
5 & 0.0907 & 17 & 0.0023 \\
6 & 0.0739 & 18 & 0.0014 \\
7 & 0.0595 & 19 & 0.0007 \\
8 & 0.0474 & 20 & 0.0004 \\
9 & 0.0372 & 21 & 0.0001 \\
10 & 0.0287 & 22 & 0.0000 \\
11 & 0.0218 & 23 & 0.0000 \\
12 & 0.0162 & 24 & 0.0000 \\
\hline
\end{tabular}

Como podemos observar, a moda dessa distribuiçào é 1, o que justifica o fato de ocorrer com certa frequência dezenas consecutivas nas extraçôes da Loto.

Aplicando-se o mesmo raciocinio para a Sena, obtemos:

$$
P(D=y)=\frac{\left(\begin{array}{c}
55-5 y \\
6
\end{array}\right)-\left(\begin{array}{c}
50-5 y \\
(
\end{array}\right)}{\left(\begin{array}{c}
50 \\
6
\end{array}\right)} \cdot 1 \leq y \leq 9
$$


cujos valores encontram-se na tabela abaixo.

Tabela 1.2 - Distribuiçâo de Probabilidades da Diferença Mínima $D$ entre os números sorteados na Sena.

\begin{tabular}{|c|c||c|c|}
\hline $\mathrm{D}$ & Probabilidade & $\mathrm{D}$ & Probabilidade \\
\hline 1 & 0.48743 & 6 & 0.00871 \\
2 & 0.27102 & 7 & 0.00212 \\
3 & 0.13940 & 8 & 0.00030 \\
4 & 0.06478 & 9 & 0.00001 \\
\cline { 2 - 4 } 5 & 0.02622 & TOTAL & 1.00000 \\
\hline
\end{tabular}

Aqui também verificamos que a moda é 1 . Neste caso a diferença para as demais probabilidades é ainda maior.

Com os dados da tabela 1.2 podemos testar a aleatoridade dos sorteios das dezenas da Sena. Observando-se os resultados até o concurso número 172, obtemos a seguinte distribuição de frequências (na última coluna estào os valores esperados obtidos, sob a hipótese de aleatoriedade, a partir da tabela 1.2): 
Tabela 1.3 - Frequências Esperadas e Observadas para a Diferença Mínima entre os números sorteados na Sena após 172 concursos.

\begin{tabular}{c|c|c}
\hline $\begin{array}{c}\text { Diferença } \\
\text { Mínima }\end{array}$ & $\begin{array}{c}\text { Frequência } \\
\text { Observada } O_{i}\end{array}$ & $\begin{array}{c}\text { Frequência } \\
\text { Esperada } E_{i}\end{array}$ \\
\hline 1 & 88 & 83.84 \\
2 & 46 & 46.62 \\
3 & 22 & 23.98 \\
4 & 11 & 11.14 \\
5 & 4 & 4.51 \\
6 & 0 & 1.50 \\
7 & 1 & 0.36 \\
8 & 0 & 0.05 \\
9 & 0 & 0.00 \\
\hline TOTAL & 172 & 172 \\
\hline
\end{tabular}

Utilizando-se o teste de Qui-Quadrado para aderência de modelos ( Mood \& Graybill \& Boes (1974)), para testarmos

$$
\begin{aligned}
H_{0}: & P(D=y) \quad \text { está de acordo com } 1.16 \\
\text { vs } & \\
H_{1}: & P(D=y) \quad \text { Nào está de acordo com } 1.16
\end{aligned}
$$

obtemos:

$$
\chi_{o b s}^{2}=\sum_{i=1}^{9} \frac{\left(O_{i}-E_{i}\right)^{2}}{E_{i}}=3.096
$$

Calculando-se o nivel descritivo, obtemos $\hat{\alpha}=P_{H_{0}} \chi_{o b s}^{2} \geq 3.096=P\left(\chi_{8}^{2} \geq 3.096\right)=$ 0.928189 .

Portanto, não rejeitamos a hipótese de que os sorteios sejam aleatórios. 


\subsection{Modelo Totalmente Aleatório.}

Consideremos a situação anterior onde temos $N$ urnas numeradas de 1 a $N$ e $r$ bolas agora distintas, por exemplo numeradas de 1 a. $r$. Como as bolas sào distintas não podemos mais utilizar o artificio de colocarmos arbitrariamente bolas nas urnas de acordo com nossas conveniências. Assim, necessitamos de outros argumentos, como veremos a seguir, para estudarmos o problema clássico da ocupação.

\section{Teorema 1.3 - Princípio da Inclusão-Exclusão}

Sejam $A_{1}, A_{2}, \ldots, A_{N}$ eventos de um mesmo espaço amostral.

Sejam $\quad p_{i}=P\left(A_{i}\right), \quad p_{i j}=P\left(A_{i} \cap A_{j}\right), \quad p_{i j k}=P\left(A_{i} \cap A_{j} \cap A_{k}\right), \ldots$

Defina $S_{0}=1, \quad S_{1}=\sum_{i} p_{i}, \quad S_{2}=\sum_{i, j} p_{i j}$ e assim sucessivamente.

Então, a probabilidade $P_{[m]}$ de que exatamente $m$ dos $N$ eventos ocorram simultaneamente é dada por:

$$
P_{[m]}=S_{m}-\left(\begin{array}{c}
m+1 \\
m
\end{array}\right) S_{m+1}+\left(\begin{array}{c}
m+2 \\
m
\end{array}\right) S_{m+2}-\cdots+(-1)^{N-m}\left(\begin{array}{l}
N \\
m
\end{array}\right) S_{N}
$$

Prova: Seja $E$ um ponto amostral pertencente a exatamente $n$ dentre os $N$ eventos. Entào $P(E)$ contribui para $P_{[m]}$ se e somente se $n=m$. Observe que $P(E)$ aparece em $S_{1}, S_{2}, \ldots, S_{n}$ mas năo aparece em $S_{n+1}, S_{n+2}, \ldots, S_{N}$.

Logo, se $n<m P(E)$ nào contribui para o termo à direita de 1.17 .

Se $n=m, P(E)$ aparece uma e apenas uma vez em $S_{m}$.

Se $n>m$, a contribuiçào de $P(E)$ no termo à direita de 1.17 é nula pois $P(E)$ aparece em $S_{k}$, para $m \leq k \leq n$. com coeficiente $\left(\begin{array}{c}n \\ k\end{array}\right)$ ( E pertence a $n$ eventos dos quais queromos 
escolher $k$ para compor $S_{k}$ ). Assim, sua contribuiçào para o termo à direita de 1.17 é:

$$
\begin{aligned}
\left(\begin{array}{l}
m \\
m
\end{array}\right)\left(\begin{array}{c}
n \\
m
\end{array}\right) & -\left(\begin{array}{c}
m+1 \\
m
\end{array}\right)\left(\begin{array}{c}
n \\
m+1
\end{array}\right)+\cdots \pm\left(\begin{array}{c}
n \\
m
\end{array}\right)\left(\begin{array}{l}
n \\
n
\end{array}\right) \\
= & \sum_{k=0}^{n-m}(-1)^{k}\left(\begin{array}{c}
m+k \\
m
\end{array}\right)\left(\begin{array}{c}
n \\
m+k
\end{array}\right) \\
= & \left(\begin{array}{c}
n \\
m
\end{array}\right) \sum_{k=0}^{n-m}(-1)^{k}\left(\begin{array}{c}
n-m \\
k
\end{array}\right) \\
= & \left(\begin{array}{c}
n \\
m
\end{array}\right)(1-1)^{n-m}=0 \quad \square
\end{aligned}
$$

Teorema 1.4 Seja $X$ o número de eventos que ocorreram dentre os $N$ eventos. Entâo o $k$-ésimo momento fatorial de $X, \mu_{X}^{(k)}$, é dado por:

$$
\mu_{X}^{(k)}=k ! S_{k}
$$

Prova: Para $i=1,2 \ldots, N$, seja

$$
I_{i}= \begin{cases}1 & \text { se } A_{i} \text { ocorre } \\ 0 & \text { caso contrário }\end{cases}
$$

Então

$$
X=\sum_{i=1}^{N} I_{i} \Longrightarrow\left(\begin{array}{c}
I_{1}+I_{2}+\cdots+I_{N} \\
k
\end{array}\right)=\sum_{1 \leq i_{1} \leq i_{2} \leq \cdots \leq i_{k} \leq N} I_{i_{1}} I_{i_{2}} \cdots I_{i_{k}}
$$

Logo,

$$
\begin{aligned}
\mathrm{E}\left[\left(\begin{array}{c}
X \\
k
\end{array}\right)\right] & =\sum_{1 \leq i_{1} \leq i_{2} \leq \cdots \leq i_{k} \leq n} \mathrm{E}\left(I_{i_{1}} I_{i_{2}} \cdots I_{i_{k}}\right)= \\
& =\sum P\left(A_{i_{1}} \cap A_{i_{2}} \cap \cdots \cap A_{i_{k}}\right)=S_{k} \\
\Longrightarrow \mu_{X}^{(k)} & =\mathrm{E}\left[X^{(k)}\right]=k ! \mathbf{E}\left[\left(\begin{array}{c}
X \\
k
\end{array}\right)\right]=k ! S_{k}
\end{aligned}
$$


Corolário 1.5 A esperança e a variância de $\mathrm{X}$ sào dadas por:

$$
\begin{aligned}
\mathbf{E}(X) & =S_{1} \\
\operatorname{Var}(X) & =2 S_{2}+S_{1}-S_{1}^{2}
\end{aligned}
$$

\subsubsection{Distribuição do número de urnas ocupadas e do número de urnas vazias}

Lema 1.4 No caso de distribuigào totalmente aleatória as distribuisós de $M_{0}^{r}$ e de $M_{\text {be }}^{r}$ são dadas por:

$$
\begin{aligned}
P\left(M_{0}^{r}=k\right) & =N^{-r}\left(\begin{array}{c}
N \\
k
\end{array}\right) \Delta^{N-k} \mathrm{O}^{r} \\
& =N^{-r} \frac{N !}{k !} \varphi_{r}^{(N-k)}, k=\max (0, N-r), \ldots, N-1 \\
P\left(M_{o c}^{r}=k\right) & =N^{-r} \frac{N !}{(N-k) !} \varphi_{r}^{(k)}, k=1, \ldots, \min (N, r)
\end{aligned}
$$

onde $\varphi_{r}^{(k)}$ sâo os números de Stirling de segunda ordem. (ver apêndice A)

Prova: Considere os eventos $A_{1}, A_{2}, \ldots, A_{N}$ definidos da seguinte maneira: $A_{i}=$ A urna $i$ está vazia, $i=1,2, \ldots, N$, e seja $p_{j}$ a probabilidade de uma bola ocupar a urna $j, j=1,2, \ldots, N$, $\operatorname{com} 0 \leq p_{j} \leq 1$ e $\sum_{j=1}^{N} p_{j}=1$.

Se todas as urnas sào igualmente prováveis. $p_{j}=\frac{1}{N} . j=1,2 \ldots, N$. Neste caso, temos que

$$
\begin{aligned}
& P\left(A_{i}\right)=\left(1-\frac{1}{N}\right)^{r} \\
& P\left(A_{i} \cap A_{j}\right)=\left(1-\frac{2}{N}\right)^{r} \\
& \vdots \\
& P\left(A_{i_{1}} \cap A_{i_{2}} \cap \cdots \cap A_{i_{k}}\right)=\left(1-\frac{k}{N}\right)^{r} \\
& \text { e } S_{k}=\left(\begin{array}{c}
N \\
k
\end{array}\right) P\left(A_{i_{1}} \cap A_{i_{2}} \cap \cdots \cap A_{i_{k}}\right)=\left(\begin{array}{c}
N \\
k
\end{array}\right)\left(\frac{N-k}{N}\right)^{r}
\end{aligned}
$$


Então por 1.17

$$
\begin{aligned}
P\left(M_{0}^{r}=k\right) & =P_{[k]}=S_{k}-\left(\begin{array}{c}
k+1 \\
k
\end{array}\right) S_{k+1}+\cdots(-1)^{N-k}\left(\begin{array}{c}
N \\
k
\end{array}\right) S_{N} \\
& =\sum_{i=0}^{N-k}(-1)^{i}\left(\begin{array}{c}
k+i \\
k
\end{array}\right) S_{k+i} \\
& =\sum_{i=0}^{N-k}(-1)^{i}\left(\begin{array}{c}
k+i \\
k
\end{array}\right)\left(\begin{array}{c}
N \\
k+i
\end{array}\right)\left(\frac{N-k-i}{N}\right)^{r} \\
& =N^{-r} \sum_{i=0}^{N-k}(-1)^{i}\left(\begin{array}{c}
k+i \\
k
\end{array}\right)\left(\begin{array}{c}
N \\
k+i
\end{array}\right)(N-k-i)^{r}
\end{aligned}
$$

$\operatorname{Mas}\left(\begin{array}{c}k+i \\ k\end{array}\right)\left(\begin{array}{c}N \\ k+i\end{array}\right)=\left(\begin{array}{c}N \\ k\end{array}\right)\left(\begin{array}{c}N-k \\ i\end{array}\right)$. Logo,

$$
\begin{aligned}
P\left(M_{0}^{r}=k\right) & =N^{-r}\left(\begin{array}{c}
N \\
k
\end{array}\right) \sum_{i=0}^{N-k}(-1)^{2}\left(\begin{array}{c}
N-k \\
i
\end{array}\right)(N-k-i)^{r} \\
& =N^{-r}\left(\begin{array}{c}
N \\
k
\end{array}\right) \Delta^{N-k} \mathrm{O}^{r} \\
& =N^{-r} \frac{N !}{k !} \varphi_{r}^{(N-k)}
\end{aligned}
$$

Como $M_{o c}^{r}=N-M_{0}^{r}$ temos que

$$
P\left(M_{o c}^{r}=k\right)=P\left(M_{0}^{r}=N-k\right)=N^{-r} \frac{N !}{(N-k) !} r_{r}^{(k)} \square
$$

\subsubsection{Momentos de $M_{0}^{r}$ e de $M_{o c}^{r}$}

Lema 1.5 A esperança e a variância de $M_{0}^{r}$ são dadas por:

$$
\begin{aligned}
\mathbf{E}\left(M_{0}^{r}\right) & =\mu_{M_{0}^{r}}^{(1)}=N^{-r} N(N-1)^{r}=N^{-(r-1)}(N-1)^{r} \\
\operatorname{Var}\left(M_{0}^{r}\right) & =N^{-(r-1)}\left[(N-1)^{r}+(N-1)(N-2)^{r}-N^{-(r-1)}(N-1)^{2 r}\right]
\end{aligned}
$$

Prova: Pelo teorema 1.4, o i-ésimo momento fatorial de $M_{0}^{r}$ é dado por:

$$
\mu_{M_{i}^{i}}^{(i)}=i ! S_{i}=i !\left(\begin{array}{c}
N \\
i
\end{array}\right)\left(\frac{N-i}{N}\right)^{r}=N^{-r} i !\left(\begin{array}{c}
N \\
i
\end{array}\right)(N-i)^{r}
$$


Portanto,

$$
\mathrm{E}\left(M_{0}^{r}\right)=\mu_{M_{0}^{r}}^{(1)}=N^{-r} N(N-1)^{r}=N^{-(r-1)}(N-1)^{r}
$$

e

$$
\begin{aligned}
\operatorname{Var}\left(M_{0}^{r}\right) & =\mu_{M_{0}^{r}}^{(2)}+\mu_{M_{0}^{r}}^{(1)}-\left[\mu_{M_{0}^{r}}^{(1)}\right]^{2} \\
& =N^{-r} 2\left(\begin{array}{c}
N \\
2
\end{array}\right)(N-2)^{r}+N^{-(r-1)}(N-1)^{r}-\left[N^{-(r-1)}(N-1)^{r}\right]^{2} \\
& =N^{-r} n(N-1)(N-2)^{r}+N^{-(r-1)}(N-1)^{r}-N^{-2(r-1)}(N-1)^{2 r} \\
& =N^{-(r-1)}\left[(N-1)^{r}+(N-1)(N-2)^{r}-N^{-(r-1)}(N-1)^{2 r}\right] \square
\end{aligned}
$$

Lema 1.6 A esperança e a variância de $M_{o c}^{r}$ são dadas por:

$$
\begin{aligned}
\mathbf{E}\left(M_{o c}^{r}\right) & =\frac{N^{r}-(N-1)^{r}}{N^{r-1}} \\
\operatorname{Var}\left(M_{o c}^{r}\right) & =\operatorname{Var}\left(M_{0}^{r}\right) \quad \text { dado por 1.24 }
\end{aligned}
$$

Prova: Para obtermos a esperança e a variância do número de urnas ocupadas, basta lembrar que $M_{o c}^{r}=N-M_{0}^{r}$. Logo,

$$
\begin{aligned}
\mathbf{E}\left(M_{o c}^{r}\right) & =N-\mathbf{E}\left(M_{0}^{r}\right)=N-\frac{(N-1)^{r}}{N^{r-1}}=\frac{N^{r}-(N-1)^{r}}{N^{r-1}} \\
\operatorname{Var}\left(M_{o c}^{r}\right) & =\operatorname{Var}\left(M_{0}^{r}\right) \square
\end{aligned}
$$

\subsubsection{Distribuição e esperança do número de urnas com exata- mente $k$ bolas}

Lema 1.7 Seja $M_{k}^{r}$ o número de urnas com exatamente $k$ bolas. Entâo,

$$
\begin{aligned}
P\left(M_{k}^{r}=j\right)= & N^{-r}\left(\begin{array}{c}
N \\
j
\end{array}\right) \frac{r !}{(k !)} \sum_{i=0}^{N-j}(-1)^{i}\left(\begin{array}{c}
N-j \\
i
\end{array}\right) \frac{(N-j-i)^{r-(j+i) k}}{(k !)^{i}(r-(j+i) k) !} \\
\mathrm{E}\left(M_{k}^{r}\right)= & N^{-(r-1)}\left(\begin{array}{c}
r \\
k
\end{array}\right)(N-1)^{r-k} \\
\operatorname{Var}\left(M_{k}^{r}\right)= & (N-1) N^{-(r-1)}\left(\begin{array}{c}
r \\
k
\end{array}\right)\left[\left(\begin{array}{c}
r-k \\
k
\end{array}\right)(N-2)^{r-2 k}\right. \\
& \left.+(N-1)^{r-k-1}+N\left(\begin{array}{c}
r \\
k
\end{array}\right)(N-1)^{2(r-k)-1}\right]
\end{aligned}
$$


Prova: Seja $E_{j}$ o evento "a urna $j$ contém exatamente $k$ bolas" e seja $X_{j}$ o número de bolas na urna $j, j=1,2, \ldots N$. Então,

$$
\begin{aligned}
X_{j} & \sim \operatorname{binomial}\left(r ; \frac{1}{N}\right) \\
\left(X_{i}, X_{j}\right) & \sim \operatorname{Multinomial}\left(r ; \frac{1}{N}, \frac{1}{N}, \frac{N-2}{N}\right) \\
& \vdots \\
\left(X_{i_{1}}, X_{i_{2}}, \ldots, X_{i_{j}}\right) & \left.\sim \text { Multinomial }\left(r ; \frac{1}{N}, \frac{1}{N}, \ldots, \frac{1}{N}, \frac{N-j}{N}\right)\right)
\end{aligned}
$$

Assim,

$$
\begin{aligned}
P\left(E_{j}\right) & =P\left(X_{j}=k\right)=\left(\begin{array}{l}
r \\
k
\end{array}\right)\left[\frac{1}{N}\right]^{k}\left[\frac{N-1}{N}\right]^{r-k}=N^{-r}\left(\begin{array}{l}
r \\
k
\end{array}\right)(N-1)^{r-k} \\
P\left(E_{i} \cap E_{j}\right) & =P\left(X_{i}=k \cap X_{i}=k\right)=N^{-r} \frac{r !}{(k !)^{2}(r-2 k) !}(N-2)^{r-2 k}
\end{aligned}
$$

$$
\begin{aligned}
P\left(E_{i_{1}} \cap E_{i_{2}} \cap \cdots \cap E_{i_{j}}\right) & =P\left(X_{i_{1}}=k \cap X_{i_{2}}=k \cap \cdots \cap X_{i_{j}}=k\right) \\
& =N^{-r} \frac{r !}{(k !)^{j}(r-k j) !}(N-j)^{r-k j}
\end{aligned}
$$

e utilizando o teorema $1.3 \mathrm{com} S_{j}$ dado por 1.31 temos:

$$
\begin{aligned}
P\left(M_{k}^{r}=j\right) & =S_{j}-\left(\begin{array}{c}
j+1 \\
j
\end{array}\right) S_{j+1}+\left(\begin{array}{c}
j+2 \\
j
\end{array}\right) S_{j+2}-\cdots \pm\left(\begin{array}{c}
N \\
j
\end{array}\right) S_{N} \\
& =\sum_{i=0}^{N-j}(-1)^{i}\left(\begin{array}{c}
j+i \\
j
\end{array}\right) S_{j+i} \\
& =\sum_{i=0}^{N-j}(-1)^{i}\left(\begin{array}{c}
j+i \\
j
\end{array}\right) N^{-r}\left(\begin{array}{c}
N \\
j+i
\end{array}\right) \frac{r^{\prime}(N-(j+i))^{r-(j+i) k}}{(k !)^{j+i}(r-(j+i) k) !} \\
& =\frac{N^{-r} r !}{(k !)^{j}} \sum_{i=0}^{N-j}(-1)^{i}\left(\begin{array}{c}
j+i \\
j
\end{array}\right)\left(\begin{array}{c}
N \\
j+i
\end{array}\right) \frac{(N-j-i)^{r-(j+i) k}}{(k !)^{i}(r-(j+i) k) !} \\
& =N^{-r}\left(\begin{array}{c}
N \\
j
\end{array}\right) \frac{r !}{(k !)^{j}} \sum_{i=0}^{N-j}(-1)^{i}\left(\begin{array}{c}
N-j \\
i
\end{array}\right) \frac{(N-j-i)^{r-(j+i) k}}{(k !)^{i}(r-(j+i) k) !}
\end{aligned}
$$

Pelo teorema 1.4. temos que:

$$
\mu_{M_{k}^{r}}^{(j)}=j ! S_{j}=N^{-r} j !\left(\begin{array}{c}
N \\
j
\end{array}\right) \frac{r !}{(k !)^{j}(r-k j) !}(N-j)^{r-k j}
$$


Logo,

$$
\begin{aligned}
\mathbf{E}\left(M_{k}^{r}\right)= & S_{1}=N\left(\begin{array}{c}
r \\
k
\end{array}\right)\left[\frac{1}{N}\right]^{k}\left[\frac{N-1}{N}\right]^{r-k}=N^{-(r-1)}\left(\begin{array}{c}
r \\
k
\end{array}\right)(N-1)^{r-k} \\
\operatorname{Var}\left(M_{k}^{r}\right)= & (N-1) N^{-(r-1)}\left(\begin{array}{c}
r \\
k
\end{array}\right)\left[\left(\begin{array}{c}
r-k \\
k
\end{array}\right)(N-2)^{r-2 k}\right. \\
& \left.+(N-1)^{r-k-1}+N\left(\begin{array}{c}
r \\
k
\end{array}\right)(N-1)^{2(r-k)-1}\right] \square
\end{aligned}
$$


Capítulo 2

O Problema das Comissões. 


\subsection{Introdução}

No capítulo 1 estudamos a distribuição de $r$ bolas em $N$ urnas que, conforme dito anteriormente, é equivalente a retirarmos $r$ bolas, uma a uma e com reposição, de uma urna contendo $N$ bolas numeradas de 1 a $N$. Neste capítulo generalizaremos os resultados do capítulo 1 , para o caso em que a cada retirada escolhemos $n$ bolas, $1 \leq n \leq N$, sem reposição dentre as $N$ bolas disponiveis.

Na literatura este caso é conhecido como Problema das Comissões (Committe Problems), que consiste no seguinte: de um grupo de $N$ pessoas devemos formar $r$ comissòes de $n$ membros cada. Qual a probabilidade de que exatamente $k$ pessoas estejam envolvidas em comissões?

Se $n=1$, estamos no problema clássico da ocupaçà visto no capítulo 1 .

Duas situações reais onde podemos aplicar os resultados deste capítulo são as loterias de números Loto e Sena. No caso da Sena temos $N=50$ e $n=6$, enquanto que para a Loto temos $N=100$ e $n=5$. 


\subsection{Distribuição e Momentos do Número de Bolas que Faltam Sair}

Lema 2.1 Seja $X_{r}$ o número de bolas que ainda não foram sorteadas nenhuma vez, após $r$ retiradas. Então, a distribuição de probabilidades de $X_{r}$ é dada por:

$$
P\left(X_{r}=k\right)=\left(\begin{array}{c}
N \\
k
\end{array}\right) \sum_{j=0}^{N-k}(-1)^{N-k-j}\left(\begin{array}{c}
N-k \\
j
\end{array}\right) \frac{\left(\begin{array}{c}
j \\
n
\end{array}\right)^{r}}{\left(\begin{array}{c}
N \\
n
\end{array}\right)^{r}} . k=\max (0, N-n r), \ldots, N-n
$$

Prova: Inicialmente observemos que a cada retirada temos $\left(\begin{array}{c}N \\ n\end{array}\right)$ maneiras diferentes de escolhermos as $n$ bolas. Assim, o número de maneiras diferentes de realizarmos retiradas de $n$ bolas cada é $\left(\begin{array}{c}N \\ n\end{array}\right)^{r}$.

Considere entâo os eventos $A_{1}^{r}, A_{2}^{r}, \ldots, A_{N}^{r}$ assim definidos:

$$
A_{i}^{r}=\text { a bola de número } i \text { nào foi retirada nenhuma vez, } i=1,2, \ldots, N \text {. }
$$

Para determinarmos a probabilidade $P\left(A_{i}^{r}\right)$ desses eventos. basta observar que para que a bola $i$ nunca seja retirada devemos escolher as bolas dentre as $N-1$ bolas que restam quando excluímos a bola de número $i$ da urna. Como isto pode ser feito de $\left(\begin{array}{c}N-1 \\ n\end{array}\right)^{r}$ maneiras diferentes, temos que

$$
P\left(A_{i}^{v}\right)=\left[\frac{\left(\begin{array}{c}
N-1 \\
n
\end{array}\right)}{\left(\begin{array}{c}
N \\
n
\end{array}\right)}\right]^{r}
$$

Do mesmo modo obtemos 


$$
\begin{aligned}
P\left(A_{i}^{r} \cap A_{j}^{r}\right)= & {\left[\frac{\left(\begin{array}{c}
N-2 \\
n
\end{array}\right)}{\left(\begin{array}{c}
N \\
n
\end{array}\right)}\right]^{r} } \\
& \vdots \\
P\left(A_{i_{1}}^{r} \cap A_{i_{2}}^{r} \cap \cdots \cap A_{i j}^{r}\right)= & {\left[\frac{\left(\begin{array}{c}
N-j \\
n
\end{array}\right)}{\left(\begin{array}{c}
N \\
n
\end{array}\right)}\right]^{r} }
\end{aligned}
$$

Deste modo, para obtermos a probabilidade de que $N-m$ bolas não sejam sorteadas nenhuma vez basta aplicar o teorema $1.3 \mathrm{com}$

$$
S_{i}=\left(\begin{array}{c}
N \\
j
\end{array}\right)\left[\frac{\left(\begin{array}{c}
N-j \\
n
\end{array}\right)}{\left(\begin{array}{c}
N \\
n
\end{array}\right)}\right]^{r}
$$

Assim,

$$
\begin{aligned}
P_{[N-m]} & =\sum_{i=0}^{N-(N-m)}(-1)^{i}\left(\begin{array}{c}
N-m+i \\
i
\end{array}\right) S_{N-m+i} \\
& =\sum_{i=0}^{m}(-1)^{i}\left(\begin{array}{c}
N-m+i \\
i
\end{array}\right)\left(\begin{array}{c}
N \\
N-m+i
\end{array}\right) \frac{\left(\begin{array}{c}
N-(N-m+i) \\
n
\end{array}\right)^{r}}{\left(\begin{array}{c}
N \\
n
\end{array}\right)^{r}} \\
& =\sum_{i=0}^{m}(-1)^{i}\left(\begin{array}{c}
N-m+i \\
i
\end{array}\right)\left(\begin{array}{c}
N-m+i
\end{array}\right) \frac{\left(\begin{array}{c}
m-i \\
n
\end{array}\right)^{r}}{\left(\begin{array}{c}
N \\
n
\end{array}\right)^{r}} \\
& =\sum_{i=0}^{m}(-1)^{i}\left(\begin{array}{c}
N \\
N-m
\end{array}\right)\left(\begin{array}{c}
m \\
i
\end{array}\right) \frac{\left(\begin{array}{c}
m-i \\
n
\end{array}\right)^{r}}{\left(\begin{array}{c}
N \\
n
\end{array}\right)^{r}}
\end{aligned}
$$




$$
=\left(\begin{array}{c}
N \\
N-m
\end{array}\right) \sum_{j=0}^{m}(-1)^{m-j}\left(\begin{array}{c}
m \\
j
\end{array}\right) \frac{\left(\begin{array}{c}
j \\
n
\end{array}\right)^{r}}{\left(\begin{array}{c}
N \\
n
\end{array}\right)^{r}}
$$

Logo por 2.4 temos que

$$
P\left(X_{r}=k\right)=P[k]=\left(\begin{array}{c}
N \\
k
\end{array}\right) \sum_{j=0}^{N-k}(-1)^{N-k-j}\left(\begin{array}{c}
N-k \\
j
\end{array}\right) \frac{\left(\begin{array}{l}
j \\
n
\end{array}\right)^{r}}{\left(\begin{array}{c}
N \\
n
\end{array}\right)^{r}}
$$

Pelo corolário 1.5 , temos que:

Lema 2.2 A esperança e variância do número de bolas que faltam sair sâo respectivamente

$$
\begin{aligned}
\mathrm{E}\left(X_{r}\right) & =N\left(1-\frac{n}{N}\right)^{r} \\
\operatorname{Var}\left(X_{r}\right) & =2 S_{2}+S_{1}-S_{1}^{2} \quad \text { onde } S_{1} \text { e } S_{2} \text { são dados por } 2.3
\end{aligned}
$$

Lema 2.3 Seja $Y_{r}$ o número de bolas que já sairam pelo menos uma vez. Então,

$$
\begin{aligned}
P\left(Y_{r}=k\right) & =P\left(X_{r}=N-k\right)=\left(\begin{array}{c}
N \\
k
\end{array}\right) \sum_{j=0}^{k}(-1)^{k-j}\left(\begin{array}{c}
k \\
j
\end{array}\right) \frac{\left(\begin{array}{c}
j \\
n
\end{array}\right)^{r}}{\left(\begin{array}{c}
N \\
n
\end{array}\right)^{r}} \\
k=n, \ldots, \min (N, n r) & \\
\mathrm{E}\left(Y_{r}\right) & =N-\mathbf{E}\left(X_{r}\right)=N\left[1-\left(1-\frac{n}{N}\right)^{r}\right] \\
\operatorname{Var}\left(Y_{r}\right) & =\operatorname{Var}\left(X_{r}\right)
\end{aligned}
$$

Prova: Como $X_{r}+Y_{r}=N$ utilizando-se 2.1 e 2.6 chegamos facilmente ao resultado. 
Capítulo 3

Problemas de Tempo de Espera. 


\subsection{Introdução}

Nos capítulos anteriores, estudamos a composição das urnas após um número fixo $r$ de ensaios terem sido realizados. Porém. em muitas situaçòes de interesse o número de ensaios a serem realizados nào é necessariamente conhecido a priori, isto é, os ensaios são realizados até que determinada condição pré-estabelecida ocorra. Nestes casos, interessamo-nos pela distribuição do número de ensaios necessários para ocorrência de tal condição. Estes problemas são chamados Problemas de Tempo de Espera (Waiting Time Problems).

A seçào 3.2 trata do problema de tempo de espera quando em cada ensaio apenas uma bola é alocada. É desenvolvida uma soluçào proposta por Holst (1986), baseada em uma imersão do problema em um Processo de Poisson.

Na seção 3.3 o problema é tratado no caso em que a cada ensaio $n$ bolas sào alocadas. Exemplo numérico aplicado à loteria de números Sena é apresentado no final da seção. 


\subsection{Tempo de Espera no Problema da Ocupação.}

\subsubsection{O Problema do Colecionador}

Considere uma urna com $N$ bolas numeradas de 1 a $N$, que são retiradas, uma por vez, com reposição. O problema do colecionador é determinar a distribuição do número mínimo de retiradas necessárias para se obter $k$ bolas distintas.

Lema 3.1 Seja $W_{k}^{N}, k \leq N$, o número mínimo de retiradas necessárias para se obter $k$ bolas distintas. Então,

$$
\begin{aligned}
P\left(W_{k}^{N}=r\right) & =N^{-r} N_{(k) i_{r-1}^{(k-1)}} \quad r=k, k+1 \ldots \\
\mathbf{E}\left(W_{N}^{N}\right) & =N \sum_{j=1}^{N} j^{-1} \\
\operatorname{Var}\left(W_{N}^{N}\right) & =N \sum_{j=1}^{N}(N-j) j^{-2}
\end{aligned}
$$

Prova: $W_{k}^{N} \leq r$ se e somente se após $r$ retiradas tivermos pelo menos $k$ bolas distintas, isto é, $M_{o c}^{r} \geq k$.

Além disso, $W_{k}^{N}=r$ se e somente se nas $r-1$ retiradas anteriores tivermos $k-1$ bolas distintas e na $r$-ésima retirada sair uma bola distinta das $k-1$ já retiradas. Portanto, utilizando 1.5 , obtemos:

$$
\begin{aligned}
P\left(W_{k}^{N}=r\right) & =P\left(M_{0 c}^{r-1}=k-1\right) P(\text { sair bola distinta das } k-1 \text { na r-ésima retirada }) \\
& =N^{-(r-1)}\left(\begin{array}{c}
N \\
k-1
\end{array}\right) \Delta^{k-1} \mathrm{O}^{r-1} \frac{N-k+1}{N} \\
& =N^{-r} N_{(k)} \frac{\Delta^{k-1} \mathrm{O}^{r-1}}{(k-1) !} \\
& =N^{-r} N_{(k)} \vartheta_{r-1}^{(k-1)}
\end{aligned}
$$


Para mostrar 3.2 e 3.3 defina $Y_{j}=$ número de retiradas entre a $j$-ésima e a $(j+1)$-ésima (inclusive) bola distinta, $j=0,1,2, \ldots$ Deste modo,

$$
\begin{aligned}
& Y_{0}=1 \\
& Y_{j} \sim \text { Geométrica }\left(1-\frac{j}{N}\right) \quad \text { independentes. }
\end{aligned}
$$

Assim,

$$
W_{k}^{N}=\sum_{j=0}^{k-1} Y_{j}
$$

Utilizando a expressào 3.4 acima, obtemos facilmente a esperança e a variância de $W_{k}^{N}$ :

$$
\begin{aligned}
\mathbf{E}\left(W_{k}^{N}\right) & =N \sum_{j=0}^{k-1}(N-j)^{-1} \\
\operatorname{Var}\left(W_{k}^{N}\right) & =N \sum_{j=0}^{k-1} j(N-j)^{-2}
\end{aligned}
$$

Corolário 3.1 Em particular, para o tempo de espera $W_{N}^{N}$ para que todas as bolas saiam pelo menos uma vez, temos:

$$
\begin{aligned}
\mathrm{E}\left(W_{N}^{N}\right) & =N \sum_{j=1}^{N} j^{-1} \\
\operatorname{Var}\left(W_{N}^{N}\right) & =N \sum_{j=1}^{N}(N-j) j^{-2}
\end{aligned}
$$


A partir de 3.4 , temos também

$$
\mathbf{E}\left[(1-t)^{-W_{k}^{N}}\right]=\mathbf{E}\left[(1-t)^{-\sum_{j=0}^{k-1} Y_{j}}\right]=\prod_{j=0}^{k-1} \mathbf{E}\left[(1-t)^{-Y_{j}}\right]
$$

Mas,

$$
\begin{aligned}
\mathbf{E}\left[(1-t)^{-\mathrm{Y} j}\right] & =\sum_{i=1}^{\infty}(1-t)^{-i}\left(1-\frac{j}{N}\right)\left(\frac{j}{N}\right)^{i-1} \\
& =\sum_{i=1}^{\infty}(1-t)^{-i} \frac{(N-j) j^{i-1}}{N^{i}} \\
& =\frac{N-j}{j} \sum_{i=1}^{\infty}\left(\frac{j}{N(1-t)}\right)^{i} \\
& =\left(1-\frac{N t}{N-j}\right)^{-1}
\end{aligned}
$$

Substituindo-se 3.8 em 3.7. obtemos:

$$
\mathbf{E}\left[(1-t)^{-W_{k}^{k}}\right]=\prod_{j=0}^{k-1}\left(1-\frac{N t}{N-j}\right)^{-1}
$$

A expressão 3.9 acima nos permite obter os momentos fatoriais ascendentes de $W_{k}^{N}$, pois

$$
\left.\frac{d^{v} \mathbf{E}\left[(1-t)^{-W_{k}^{N}}\right]}{d t^{v}}\right|_{t=0}=\mathbf{E}\left[W_{k}^{N}\left(W_{k}^{N}+1\right) \ldots\left(W_{k}^{N}+v-1\right)\right]
$$




\subsubsection{Imersão em um Processo de Poisson}

Os problemas de tempo de espera relacionados com o problema clássico da ocupação, podem ser estudados via uma imersâo em um Processo de Poisson, da seguinte forma: toda vez que ocorrer um evento no processo, retira-se uma bola da urna e marca-se esse evento com o número da bola selecionada. Demonstra-se que com isso, obtêm-se um conjunto de Processos de Poisson independentes (Ross (1985)), cada um associado ao número da bola correspondente. Deste modo, o número de retiradas necessárias para que determinadas condiçôes se verifiquem coincide com os tempos de espera para a ocorrência de eventos nos Processos de Poisson correspondentes.

Teorema 3.2 Sejam $T_{1}, T_{2}, \ldots, T_{N}$ variáveis aleatórias independentes com $T_{j} \sim \Gamma\left(m_{j} ; p_{j}\right)$ e $T_{1}^{N}, T_{2}^{N}, \ldots, T_{N}^{N}$ suas estatisticas de ordem. Para thsaios multinomiais com probabilidades $p_{1}, p_{2}, \ldots, p_{N}$ e quotas $m_{1}, m_{2}, \ldots, m_{N}$. seja $W_{k}^{N}$ o número de ensaios até que $k$ quotas estejam satisfeitas. Entào, para $t<\min \left(p_{j}\right)$.

$$
\begin{gathered}
\mathrm{E}\left[(1-t)^{-W_{k}^{N}}\right]=\mathrm{E}\left[e^{t T_{k}^{N}}\right] \\
\mathrm{E}\left[W_{k}^{N}\left(W_{k}^{N}+1\right) \ldots\left(W_{k}^{N}+\imath-1\right)\right]=\mathbf{E}\left[\left(T_{k}^{N}\right)^{v}\right]
\end{gathered}
$$

Prova: Seja $\mathcal{P}$ um processo de Poisson com intensidade $\lambda=1$ e sejam $Z_{1}, Z_{2}, \ldots$ os tempos entre as ocorrências. Então $Z_{1}, Z_{2}, \ldots$ são variáveis aleatórias independentes e identicamente distribuídas com distribuição exponencial de parâmetro 1 .

Para cada ocorrência no processo $\mathcal{P}$. associamos uma marca $I$ de modo que as marcas sejam independentes e identicamente distribuídas, $\operatorname{com} P(I=j)=p_{j}, j=1,2, \ldots, N$, e sejam independentes dos tempos entre as ocorrências. Deste modo, as ocorrências marcadas com $I=j$ definem um processo de Poisson $\mathcal{P}_{j}$ com intensidade $p_{j}$ e os processos resultantes $\mathcal{P}_{1}, \mathcal{P}_{2}, \ldots \mathcal{P}_{N}$ são independentes.

Definição 3.1 Divemos que o processo $\mathcal{P}_{j}$ completou sua quota, quando tivermos pelo menos $m_{j}$ ocorrências em $\mathcal{P}_{j}$. O número $m_{j}$ será chamado quota do processo $\mathcal{P}_{j}$. 
Seja $T_{j}$ o tempo necessário para o processo $\mathcal{P}_{j}$ completar sua quota. Entào $T_{j} \sim \Gamma\left(m_{j} ; p_{j}\right)$, isto é,

$$
f_{T_{j}}(t)=\frac{p_{j}^{m_{j}}}{\Gamma\left(m_{j}\right)} t^{m_{j}-1} e^{-p_{j} t}=\frac{p_{j}^{m_{j}}}{\left(m_{j}-1\right) !} t^{m_{j}-1} e^{-p_{j} t}, \quad t \geq 0
$$

Assim, o tempo de espera $T_{k}^{N}$ até que $k$ processos completem suas quotas é a $k$-ésima estatística de ordem de $T_{1}, T_{2}, \ldots, T_{N}$.

Seja $W_{k}^{N}$ o número total de ocorrências no processo $\mathcal{P}$ no instante $T_{k}^{N}$. Então,

$$
T_{k}^{N}=\sum_{i=1}^{W_{k}^{N}} Z_{i}
$$

Logo, para $t<\min \left(p_{j}\right)$ temos:

$$
\begin{aligned}
\mathbf{E}\left(\epsilon^{t T_{k}^{N}}\right) & =\mathbf{E}\left(\epsilon^{t \sum_{i=1}^{W_{k}^{N}} Z_{1}}\right) \\
& =\mathbf{E}\left[\mathbf{E}\left(\epsilon^{t \sum_{i=1}^{W_{k}^{N}} Z_{i}} \mid W_{k}^{N}\right)\right] \\
& =\mathbf{E}\left[\mathbf{E}\left(\prod_{i=1}^{W_{k}^{N}} \epsilon^{t Z_{i}} \mid W_{k}^{N}\right)\right] \\
& =\mathbf{E}\left[\mathbf{E}\left(\left(\epsilon^{t Z_{1}}\right)^{W_{k}^{N}} \mid W_{k}^{N}\right)\right] \\
& =\mathbf{E}\left[(1-t)^{-1 W_{k}^{N}}\right]
\end{aligned}
$$

Da expressào acima, segue que os momentos fatoriais ascendentes de $W_{k}^{N}$ satisfaz:

$$
\mathbf{E}\left[W_{k}^{N}\left(W_{k}^{N}+1\right) \ldots\left(W_{k}^{N}+v-1\right)\right]=\mathbf{E}\left[\left(T_{k}^{N}\right)^{v}\right]
$$

o que completa a prova do teorema. 


\subsubsection{Aplicações}

1- "The Dixie Cup Problem"

Quantas retiradas sào necessárias para que todas as $N$ bolas sejam obtidas pelo menos $m$ vezes cada?

Neste caso, as quotas de cada bola sào todas iguais a $m$, isto é, $m_{1}=m_{2}=\cdots=m_{N}=m$ e sendo as bolas igualmente prováveis de serem obtidas. $p_{1}=p_{2}=\cdots=p_{N}=\frac{1}{N}$, de modo que os correspondentes processos de Poisson tem todos intensidade $\frac{1}{N}$ e quotas $m$.

O tempo de espera $T_{j}$ para que o processo $\mathcal{F}_{j}$ complete suas $m$ ocorrências, tem distribuição $\Gamma\left(m ; \frac{1}{N}\right)=N \Gamma(m: 1)$. Logo.

$$
T_{i}=N S_{j} \text { onde } S_{j} \sim \Gamma(m ; 1)
$$

Deste modo, o tempo de espera $T_{k}^{N}$ até que pela primeira vez, todos os processos tenham ocorrido pelo menos m vezes cada será:

$$
\begin{aligned}
T_{N}^{N} & =\max \left(T_{1}, T_{2} \ldots T_{N}\right) \\
& =\max \left(N S_{1}, N S_{2} \ldots N S_{N}\right) \\
& =N \max \left(S_{1}, S_{2}, \ldots S_{N}\right) \\
& =N S_{N}^{N}
\end{aligned}
$$

Seja $W_{N}^{N}$ o número total de ocorrências, isto é. o número de retiradas. Entào.

$$
T_{N}^{N}=N S_{N}^{N}=\sum_{i=1}^{W_{N}^{N}} Z_{i} \text { onde } Z_{i} \sim \text { Exponencial(1) }
$$

Assim, pelo teorema 3.2 temos para. $1<\frac{1}{N}$ 


$$
\mathbf{E}\left[(1-t)^{-W_{N}^{N}}\right]=\mathbf{E}\left(e^{t N S_{N}^{N}}\right)
$$

e

$$
\mathbf{E}\left[W_{N}^{N}\left(W_{N}^{N}+1\right) \ldots\left(W_{N}^{N}+v-1\right)\right]=N^{v} \mathbf{E}\left[\left(S_{N}^{N}\right)^{v}\right]
$$

Em 3.13 fazendo $v=1$ temos

$$
\begin{aligned}
\mathrm{E}\left(W_{N}^{N}\right) & =N \mathrm{E}\left(S_{N}^{N}\right) \\
& =N \int_{0}^{\infty} P\left(S_{N}^{N}>t\right) d t \\
& =N \int_{0}^{\infty}\left[1-P\left(S_{N}^{N} \leq t\right)\right] d t \\
& =N \int_{0}^{\infty}\left[1-\left(P\left(S_{1} \leq t\right)\right)^{N}\right] d t \\
& =N \int_{0}^{\infty}\left[1-\left(1-P\left(S_{1}>t\right)\right)^{N}\right] d t \\
& =N \int_{0}^{\infty}\left[1-\left(1-\sum_{i=0}^{m-1} \frac{t^{-t} f^{j}}{j !}\right)^{N}\right] d t
\end{aligned}
$$

No caso particular em que $m=1$ (seçào 3.2.1).

$$
\begin{aligned}
\mathbf{E}\left(W_{N}^{N}\right) & =N \int_{0}^{\infty}\left[1-\left(1-\epsilon^{-t}\right)^{N}\right] d t \\
& =N \int_{0}^{\infty}\left[1-\sum_{k=0}^{N}(-1)^{k}\left(\begin{array}{c}
N \\
k
\end{array}\right) \epsilon^{-k t}\right] d t \\
& =N \int_{0}^{\infty}\left[1-1-\sum_{k=1}^{N}(-1) k\left(\begin{array}{c}
N \\
k
\end{array}\right) \epsilon^{-k t}\right] d t \\
& =N \int_{0}^{\infty} \sum_{k=1}^{N}(-1)^{k-1}\left(\begin{array}{c}
N \\
k
\end{array}\right) \epsilon^{-k t} d t \\
& =N \sum_{k=1}^{N}(-1)^{k-1}\left(\begin{array}{c}
N \\
k
\end{array}\right) \int_{0}^{\infty} \epsilon^{-k t} d t \\
& =N \sum_{k=1}^{N}(-1)^{k-1}\left(\begin{array}{c}
N \\
k
\end{array}\right) k^{-1}
\end{aligned}
$$


Por indução em $N$ é fácil mostrar que 3.15 é igual a 3.5 obtida anteriormente.

2- O Problema dos Aniversários

Quantas pessoas sào necessárias para que $m$ aniversariem no mesmo dia?

Aqui também o tempo de espera $T_{j}$ para que o processo $\mathcal{P}_{j}$ complete sua quota $(m$ ocorrências) tem distribuiçâo $\Gamma\left(m ; \frac{1}{N}\right)=N \Gamma(m ; 1)$. Estamos porém interessados na distribuiçào de $T_{1}^{N}$.

$$
T_{1}^{N}=\min \left(T_{1}, T_{2} \ldots, T_{N}\right)=N \min \left(S_{1}, S_{2} \ldots S_{N}\right)=N S_{1}^{N} \text { onde } S_{i} \sim \Gamma(m ; 1)
$$

Pelo teorema 3.2 , sendo $W_{1}^{N}$ o número de ocorrências no instante $T_{1}^{N}$, temos:

$$
\begin{aligned}
\mathbf{E}\left(W_{1}^{N}\right) & =N \mathbf{E}\left(S_{1}^{N}\right) \\
& =N \int_{0}^{\infty} P\left(S_{1}^{N}>t\right) d t \\
& =N \int_{0}^{\infty}\left(P\left(S_{1}>t\right)\right)^{N} d t \\
& =N \int_{0}^{\infty}\left[\sum_{k=0}^{m-1} \frac{t^{-t} t^{k}}{k !}\right]^{N} d t
\end{aligned}
$$

No caso $m=2$. isto é, quantas pessoas sào necessárias para que duas aniversariem no mesmo dia. temos:

$$
\begin{aligned}
\mathbf{E}\left(W_{1}^{N}\right) & =N \int_{0}^{\infty}\left(\sum_{k=0}^{1} \frac{t^{-t} t^{k}}{k !}\right)^{N} d t \\
& =N \int_{0}^{\infty}\left(t^{-t}+t t-t\right)^{N} d t \\
& =N \int_{0}^{\infty}(t+1)^{N} \epsilon^{-N t} d t
\end{aligned}
$$




$$
\begin{aligned}
& =N \int_{1}^{\infty} x^{N} e^{-N(x-1)} d x \\
& =N \epsilon^{N} \int_{1}^{\infty} x^{N} e^{-N x} d x \\
& =N e^{N}\left[\frac{e^{-N}}{N} \sum_{k=0}^{N} \frac{N_{(k)}}{N^{k}}\right] \\
& =\sum_{k=0}^{N} \frac{N(k)}{N^{k}}
\end{aligned}
$$

Considerando o ano $\operatorname{com} N=365$ dias, temos

$$
\mathbf{E}\left(W_{1}^{365}\right)=\sum_{k=0}^{365} \frac{(365)_{k}}{(365)^{k}}=24,6166
$$

Portanto, em média sào necessárias 24,6166 pessoas para que duas façam aniversário no mesmo dia. 


\subsection{Tempo de Espera no Problema das Comissões.}

Lema 3.2 Seja $T_{j}$ o número de retiradas até que j números especificos saiam pelo menos uma vez cada. Entào,

$$
\begin{aligned}
P\left(T_{j}=r\right) & =\sum_{k=1}^{j}(-1)^{k-1}\left(\begin{array}{l}
j \\
k
\end{array}\right) P_{k}^{r-1}\left(1-p_{k}\right), r=\left[\frac{j}{n}\right]+1, \ldots \\
\mathbf{E}\left(T_{j}\right) & =\sum_{k=1}^{j}(-1)^{k-1}\left(\begin{array}{l}
j \\
k
\end{array}\right)\left(1-p_{k}\right)^{-1} \\
\mathbf{E}\left(T_{j}^{2}\right) & =\sum_{k=1}^{j}(-1)^{k-1}\left(\begin{array}{l}
j \\
k
\end{array}\right) \frac{1+p_{k}}{\left(1-p_{k}\right)^{2}}
\end{aligned}
$$

onde $[k]$ indica o maior inteiro contido $\mathrm{em} k$.

Prova: Considere os eventos $A_{1}^{r}, A_{2}^{r}, \ldots, A_{N}^{r}$ como definidos na seçào 2.2 , isto é,

$$
A_{i}^{r}=\text { a bola de número } i \text { nào foi retirada nenhuma vez nas } r \text { primeiras retiradas. }
$$

Entào,

$$
\begin{aligned}
& P\left(A_{i}^{r}\right)=\left[\frac{\left(\begin{array}{c}
N-1 \\
n
\end{array}\right)}{\left(\begin{array}{c}
N \\
n
\end{array}\right)}\right]^{r}=p_{1}^{r} \\
& P\left(A_{i}^{r} \cap A_{j}^{r}\right)=\left[\frac{\left(\begin{array}{c}
N-2 \\
n
\end{array}\right)}{\left(\begin{array}{c}
N \\
n
\end{array}\right)}\right]^{r}=p_{2}^{r} \\
& \vdots\left(A_{i_{1}}^{r} \cap A_{i_{2}}^{r} \cap \cdots \cap A_{i_{j}}^{r}\right)= \\
& {\left[\frac{\left(\begin{array}{c}
N-j \\
n
\end{array}\right)}{\left(\begin{array}{c}
N \\
n
\end{array}\right)}\right]^{r}=p_{j}^{r} }
\end{aligned}
$$

Observe que $\left\{T_{j}>r\right\} \Longleftrightarrow\{$ pelo menos um dos $j$ números especificos não sair nenhuma vez após $r$ retiradas sucessivas $\}$, isto é, 


$$
\left\{T_{j}>r\right\}=\bigcup_{k=1}^{j} A_{i_{k}}^{r}
$$

Logo,

$$
P\left(T_{j}>r\right)=P\left(\bigcup_{k=1}^{j} A_{i_{k}}^{r}\right)=\sum_{k=1}^{j}(-1)^{k-1}\left(\begin{array}{l}
j \\
k
\end{array}\right) p_{k}^{r}
$$

Para obtermos a distribuiçào de $T_{j}$, basta lembrar que

$$
P\left(T_{j}=r\right)=P\left(T_{j}>r-1\right)-P\left(T_{j}>r\right)
$$

Substituindo-se 3.21 na expressào acima, obtemos:

$$
P\left(T_{j}=r\right)=\sum_{k=1}^{j}(-1)^{k-1}\left(\begin{array}{l}
j \\
k
\end{array}\right) P_{k}^{r-1}\left(1-p_{k}\right)
$$

Observe que a distribuiçâo de $T_{j}$ é uma combinação linear de $j$ distribuiçôes geométricas. Com isso, podemos obter os momentos de $T_{j}$ através dos momentos de distribuições geométricas. Logo,

$$
\mathbf{E}\left(e^{s T_{\jmath}}\right)=\sum_{k=1}^{j}(-1)^{k=1}\left(\begin{array}{l}
j \\
k
\end{array}\right) \frac{\left(1-p_{k}\right) e^{s}}{1-p_{k} e^{s}}
$$

De 3.22 obtemos:

$$
\begin{aligned}
& \mathbf{E}\left(T_{j}\right)=\sum_{k=1}^{j}(-1)^{k-1}\left(\begin{array}{l}
j \\
k
\end{array}\right)\left(1-p_{k}\right)^{-1} \\
& \mathbf{E}\left(T_{j}^{2}\right)=\sum_{k=1}^{j}(-1)^{k-1}\left(\begin{array}{l}
j \\
k
\end{array}\right) \frac{1+p_{k}}{\left(1-p_{k}\right)^{2}}
\end{aligned}
$$




\subsubsection{Aplicação.}

No caso da Sena, temos $N=50$ dezenas das quais a Caixa Econômica Federal sorteia semanalmente $n=6$ dezenas. A tabela 3.1 abaixo apresenta a média e o desvio-padrão de $T_{j}$

Tabela 3.1 - Média e Desvio-padrão de $T_{j}$

\begin{tabular}{|c|c|c||c|c|c|}
\hline $\mathrm{j}$ & Média & Desvio-padrào & $\mathrm{j}$ & Média & Desvio-padrào \\
\hline 1 & 8.3333 & 7.8174 & 26 & 30.8912 & 9.7706 \\
2 & 12.2760 & 8.7319 & 27 & 31.1841 & 9.7725 \\
3 & 14.9117 & 9.0966 & 28 & 31.4664 & 9.7741 \\
4 & 16.8883 & 9.2911 & 29 & 31.7391 & 9.7756 \\
5 & 18.4696 & 9.4107 & 30 & 32.0026 & 9.7769 \\
6 & 19.7873 & 9.4909 & 31 & 32.2577 & 9.7780 \\
7 & 20.9168 & 9.5480 & 32 & 32.5047 & 9.7791 \\
8 & 21.9051 & 9.5904 & 33 & 32.7443 & 9.7800 \\
9 & 22.7836 & 9.6230 & 34 & 32.9769 & 9.7807 \\
10 & 23.5742 & 9.6486 & 35 & 33.2028 & 9.7814 \\
11 & 24.2930 & 9.6691 & 36 & 33.4224 & 9.7820 \\
12 & 24.9519 & 9.6858 & 37 & 33.6361 & 9.7825 \\
13 & 25.5600 & 9.6997 & 38 & 33.8441 & 9.7830 \\
14 & 26.1248 & 9.7113 & 39 & 34.0469 & 9.7833 \\
15 & 26.6519 & 9.7210 & 40 & 34.2446 & 9.7835 \\
16 & 27.1460 & 9.7293 & 41 & 34.4374 & 9.7839 \\
17 & 27.6111 & 9.7365 & 42 & 34.6256 & 9.7840 \\
18 & 28.0504 & 9.7426 & 43 & 34.8095 & 9.7841 \\
19 & 28.4665 & 9.7479 & 41 & 34.9896 & 9.7828 \\
20 & 28.8618 & 9.7526 & 45 & 35.1644 & 9.7863 \\
21 & 29.2383 & 9.7566 & 46 & 35.3363 & 9.7862 \\
22 & 29.5977 & 9.7602 & 47 & 35.5061 & 9.7806 \\
23 & 29.9414 & 9.7633 & 48 & 35.6709 & 9.7801 \\
24 & 30.2709 & 9.7660 & 49 & 35.8304 & 9.7874 \\
25 & 30.5871 & 9.7684 & 50 & 35.9812 & 9.8131 \\
\hline
\end{tabular}

Observando-se os resultados da Sena até o concurso 176, e reiniciando-se a contagem toda vez que todas as 50 dezenas ocorram, verificamos que isso aconteceu três vezes, nos concursos de números 29, 69 e 129. Logo, tivemos três observações de $T_{50}$, a saber, 29,40 e 60. Para essas observaçòes, o número médio de concursos foi de 43 , pouco superior (cerca de 0,7 desvios-padrão) à média de $T_{50}$, que é de 35,98 concursos. 
É interessante observar que após o concurso 176 , estava faltando sair apenas a dezena 50 (cuja última ocorrência tinha sido no concurso 126), para que tivéssemos nova observação de $T_{50}$

\subsubsection{Conclusões e Comentários.}

A técnica de imersâo de problemas de tempo de espera em um Processo de Poisson permite a obtençào dos momentos do número mínimo de retiradas necessárias para que uma determinada condição esteja satisfeita. A sua utilizaçào no entanto está limitada ao caso em que a cada retirada uma única bola é selecionada. Quando em cada retirada mais de uma bola é selecionada, os processos $\mathcal{P}_{j}$ nâo sào mais independentes. Um problema interessante que poderia ser considerado é a procura de uma técnica que permitisse o tratamento do problema para retiradas múltiplas.

O tempo de espera até que determinada condiçâo ocorra, decresce a medida que aumentamos o número de bolas selecionadas a cada retirada, isto é, quanto maior o número de bolas selecionadas, menor o tempo de espera. Se supormos que o custo de cada retirada é uma funçâo do número de bolas selecionadas, um outro problema a ser considerado é a determinação da "estratégia ótima", no sentido de determinar o número de bolas a serem selecionadas a cada retirada que propicie o menor custo com tempo de espera limitado.

As distribuiçôes exatas das variáveis estudadas neste trabalho nâo são apresentadas sob a forma de expressòes fechadas. Uma alternativa possivel seria considerar a proximações para essas distribuiçôes, especialmente no caso em que o número de bolas e urnas for grande, quando distribuiçôes assintóticas fornecem resultados bastante satisfatórios. 
Apêndice A

Operador Diferença e Números de Stirling. 
Definição A.1 Dada uma funçâo $f$, chama-se Translaçâo de ordem $n$ de $f$ à funçâo

$$
E^{n} f(x)=f(x+n)
$$

Definição A.2 Dada uma funçâo $f$, chama-se diferença primeira de $f$, à funçâoo

$$
\Delta f(x)=f(x+1)-f(x)=(E-1) f(x)
$$

Se $n$ é um inteiro positivo, entào a diferença de ordem $n$ de $f$ é a função

$$
\Delta^{n} f(x)=(E-1) f(x)=\sum_{j=0}^{n}(-1)^{j}\left(\begin{array}{c}
n \\
j
\end{array}\right) f(x+n-j)
$$

Fazendo-se $f(x)=x^{s}$ na expressão acima obtemos:

$$
\Delta^{n} x^{s}=\sum_{j=0}^{n}(-1)^{j}\left(\begin{array}{l}
n \\
j
\end{array}\right)(x+n-j)^{s}
$$

Definição A.3 Chamamos Diferenga de Zero ao valor

$$
\Delta^{n} \mathrm{O}^{s}=\left.\Delta^{n} x^{s}\right|_{x=0}=\sum_{j=0}^{n}(-1)^{j}\left(\begin{array}{c}
n \\
j
\end{array}\right)(n-j)^{s}
$$

Definição A.4 Chama-se Número de Stirling de segunda ordem ao valor

$$
\varphi_{s}^{(n)}=\frac{\Delta^{n} \mathrm{O}^{s}}{n !}
$$


As diferença de Zero e os números de Stirling de segunda ordem apresentam as seguintes propriedades:

(i) $\Delta^{n} \mathrm{O}^{s+1}=n\left\{\Delta^{n} \mathrm{O}^{s}+\Delta^{n-1} \mathrm{O}^{s}\right\}$

(ii) $\varphi_{s}^{(n)}=0$ se $n>s$

(iii) $\varphi_{s}^{(1)}=\varphi_{s}^{(s)}=1$

(iv) $\varphi_{s+1}^{(n)}=n \varphi_{s}^{(n)}+\varphi_{s}^{(n-1)}$

Tabela A.1 - Números de Stirling de Segunda Ordem $\varphi_{s}^{(n)}$.

\begin{tabular}{r|rrrrrrrr}
\hline & \multicolumn{1}{|c}{$\mathrm{n}$} \\
\cline { 2 - 9 } & \multicolumn{1}{|c}{3} & 3 & 4 & 5 & 6 & 7 & 8 & 9 \\
\hline 2 & 1 & & & & & & & \\
3 & 3 & 1 & & & & & & \\
4 & 7 & 6 & 1 & & & & & \\
5 & 15 & 25 & 10 & 1 & & & & \\
6 & 31 & 90 & 65 & 15 & 1 & & & \\
7 & 63 & 301 & 350 & 140 & 21 & 1 & & \\
8 & 127 & 966 & 1701 & 1050 & 266 & 28 & 1 & \\
9 & 255 & 3025 & 7770 & 6951 & 2646 & 462 & 36 & 1 \\
10 & 511 & 9330 & 34105 & 42525 & 22827 & 5880 & 750 & 45 \\
\hline
\end{tabular}




\section{Bibliografia}

[1] Feller, William (1968) - An Introduction to Probability Theory and its Applications Vol. I, 3rd. edition - John Wiley \& Sons, New York.

[2] Gittelsohn, Alan M. (1969) - An Occupancy Problem - The American Statistician, April, $11-12$.

[3] Holst, Lars (1986) - On Birthday, Collectors', Occupancy and Other Classical Urn Problems - International Statistical Review, 54,1.15-27.

[4] Johnson, Norman L. \& Kotz. Samuel (1977) - Um Models and Their Application. An Approach to Modern Discrete Probability Theory - John Wiley \& Sons, New York.

[5] Leite, J. G. \& Pereira, C. A. B. \& Rodrigues, F. W. (1991) - Wainting Times to Exhaust Lottery Number - Relatório Técnico RT-MAE-9120 (IME-USP).

[6] Mantel, Nathan \& Pasternack, Bernard S. (1968) - A Class of Occupancy Problems The American Statistician, April, 23-24.

[7] Margenau, H. \& Murphy, G. M. (1943) - The Mathematics of Physics and Chemistry Van Nostrand, New York.

[8] Mood, Alexander M. \& Graybill, Franklin A. \& Boes, Duane C. (1974) - Introduction to the Theory of Statistics, 3rd. edition - Mc Graw Hill, New York.

[9] Rodrigues, F. W. \& Wechsler, S. (1982) - A Distância Mínima Entre Números Sorteados ao Acaso - Comunicaçào apresentada no V-SINAPE Simpósio Nacional de Probabilidade e Estatistica.

[10] Ross, Sheldon M. (1985) - Introduction to Probability Models - Academic Press, New York

[11] Sprott, D. A. (1969) - A Note on A Class of Occupancy Problems - The American Statistician, April, 12-13.

[12] White, Colin (1971) - The Committee Problem - The American Statistician, October, $25-26$. 